\title{
The Hidden Variable: A Case of Dasatinib- Induced Respiratory Failure
}

Dimitrios Drekolias ${ }^{1}$, Naga Vaishnavi Gadela ${ }^{2}$, Asma Syeda ${ }^{1}$, Jason Jacob ${ }^{3}$

1. Internal Medicine, University of Connecticut Health, Farmington, USA 2. Internal Medicine, University of Connecticut, Farmington, USA 3. Internal Medicine, Hartford Hospital, Hartford, USA

Corresponding author: Dimitrios Drekolias, drekolias@uchc.edu

\begin{abstract}
Tyrosine kinase inhibitors that target the BCR/ABL mutation have been used as therapies of BCR/ABL positive acute lymphoblastic leukemia (ALL) with significant results. Dasatinib is a multitargeted tyrosine kinase inhibitor with significant activity in Philadephia positive ALL which is resistant to imatinib, as well as in treatment-naïve patients. We present a case of an elderly patient with Philadelphia chromosome-positive ALL, who presented with acute hypoxic respiratory failure in the setting of active immunotherapy with dasatinib.
\end{abstract}

Categories: Internal Medicine, Oncology, Pulmonology

Keywords: acute lymphoblastic leukemia (all), philadelphia chromosome, dasatinib, respiratory failure

\section{Introduction}

Acute lymphoblastic leukemia (ALL) is primarily a disease of the pediatric population affecting mainly ages younger than six years. There is a second epidemiological peak in adults older than 60 years $[1,2]$. The annual incidence of ALL is approximately 1-5/100,000. More than $60 \%$ of the cases are of the B-cell phenotype. It has been noted that B-ALL is occurring slightly more frequently in males compared to females. The Hispanic population appears to have the highest incidence among ethnic groups and the White population is affected approximately three times more than the African American population [3]. The $\mathrm{BCR} / \mathrm{ABL}$ translocation is infrequent in ALL. The tyrosine kinase inhibitors targeting this mutation have been used in the treatment of BCR/ABL positive ALL cases. We present a case of an elderly patient with Philadelphia chromosome-positive ALL, who presented with acute hypoxic respiratory failure in the setting of active immunotherapy with the tyrosine kinase inhibitor, dasatinib.

Review began $11 / 19 / 2020$ Review ended 11/29/2020 Published 12/04/2020

\section{() Copyright 2020}

Drekolias et al. This is an open access article distributed under the terms of the Creative Commons Attribution License CC-BY 4.0., which permits unrestricted use, distribution, and reproduction in any medium, provided the original author and source are credited.

\section{Case Presentation}

A 78-year-old male patient with a history of a recent diagnosis of acute lymphoblastic leukemia on immunotherapy, prostate cancer, lower extremity deep venous thrombosis with inferior vena cava filter, and hypertension, presented to the emergency department after developing progressively worsening dyspnea, cough, and fever of approximately one-week duration.

The patient had a recent admission approximately two months prior to this presentation. At that time, he endorsed loss of appetite and weight loss, accompanied by significant leukocytosis (white blood cell count of $72,900 / \mathrm{uL}$ ) and thrombocytopenia (platelet count of 34,000/uL). Peripheral smear was suggestive of ALL. Flow cytometry revealed approximately $85-90 \%$ blasts, which expressed B-cell lineage markers (positive for HLA-DR, TdT, CD10, CD34, C19, CD20, CD22, and CD79a), consistent with the diagnosis of B-cell ALL. Cytogenetic analysis was also obtained and revealed the BCR/ABL translocation (the Philadelphia chromosome) as well as 9p21 homozygous deletion. He was subsequently started on the tyrosine kinase inhibitor, dasatinib.

During this admission, the patient denied any recent sick contacts and reported a recent COVID-19 negative test. In the emergency department, he was febrile to $102.9^{\circ} \mathrm{F}$, tachycardic to 143 , tachypneic with $22-34$ breaths/min, maintaining oxygen saturation of $93 \%$ on $6 \mathrm{~L}$ nasal cannula supplementation. The physical examination did not reveal any signs of volume overload with no crackles and no jugular venous distention or lower extremity edema present. Chest x-ray was obtained given concern for pneumonia, with haziness and streaky densities at the right lung base noted, concerning for atelectasis. Left basilar linear densities were also present, favoring atelectasis. However, no opacities were noted (Figure 1). CT angiography of the chest (CTA of the chest) was obtained for pulmonary embolism evaluation and revealed bilateral pleural effusions with no signs of any inflammatory process or pulmonary embolism (Figure 2). The patient was initially placed on broad-spectrum antibiotics with de-escalation to ceftriaxone and doxycycline. Bilateral thoracentesis was performed draining approximately 800cc. The pleural fluid analysis was consistent with transudate. Pleural fluid cytology was negative for malignant cells. The rest of the infectious workup was negative, including negative urinalysis and negative blood cultures. COVID-19 test was negative. Procalcitonin was $0.21 \mathrm{ng} / \mathrm{mL}$. Troponin was negative. Transthoracic echocardiogram was also obtained revealing biatrial dilation and a left ventricular ejection fraction of $69 \%$. Given the absence of 


\section{Cureus}

apparent etiology for the development of the pleural effusions, the decision was made to hold dasatinib. Subsequently, the patient was able to be weaned off oxygen supplementation to room air. He remained afebrile with no subsequent development of pleural effusions and was switched to nilotinib for management of his acute lymphoblastic leukemia.

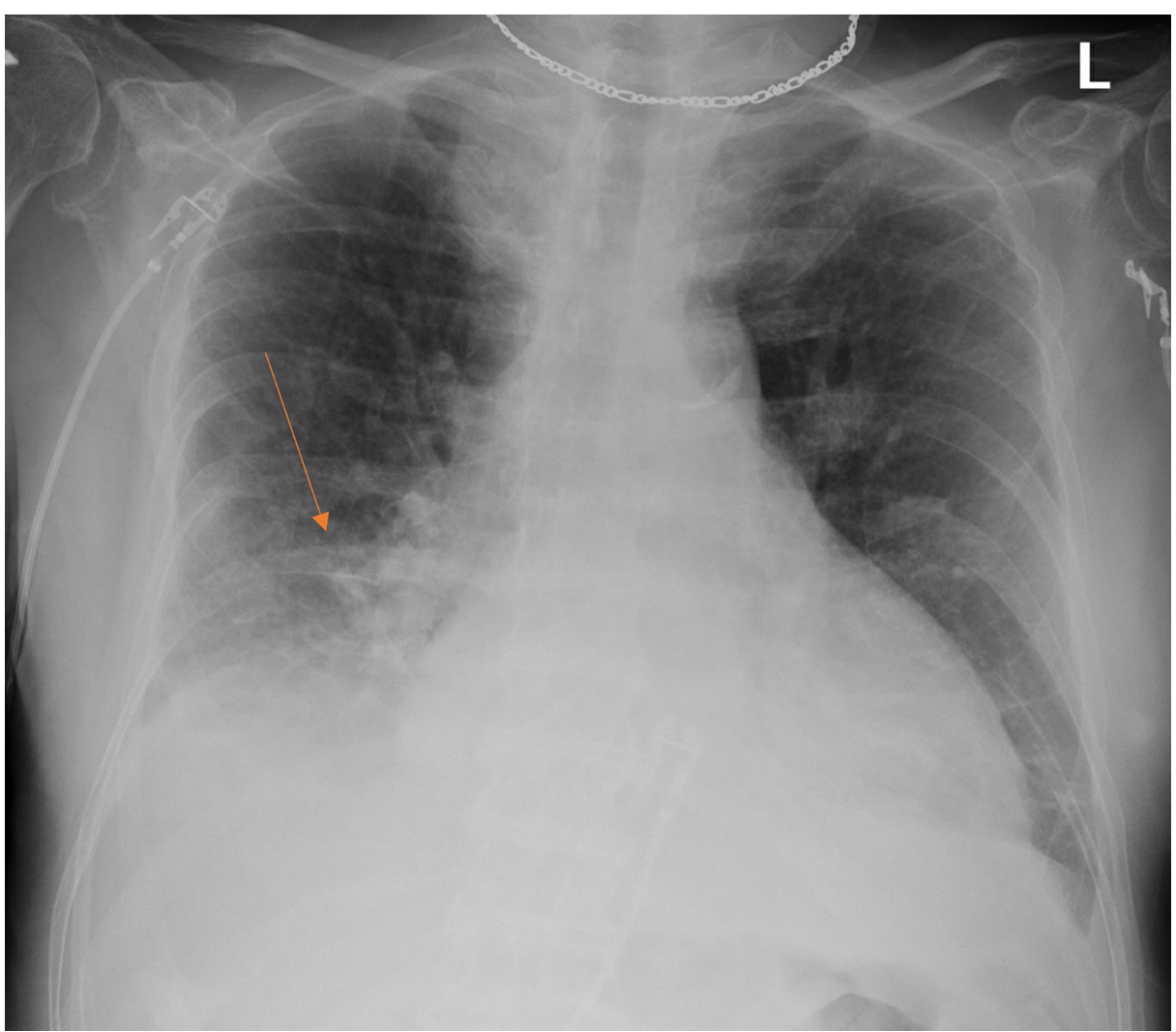

FIGURE 1: Chest x-ray, with haziness and streaky densities at the right lung base noted (orange arrow), concerning atelectasis. Left basilar linear densities also present, favoring atelectasis. However, no opacities were noted.

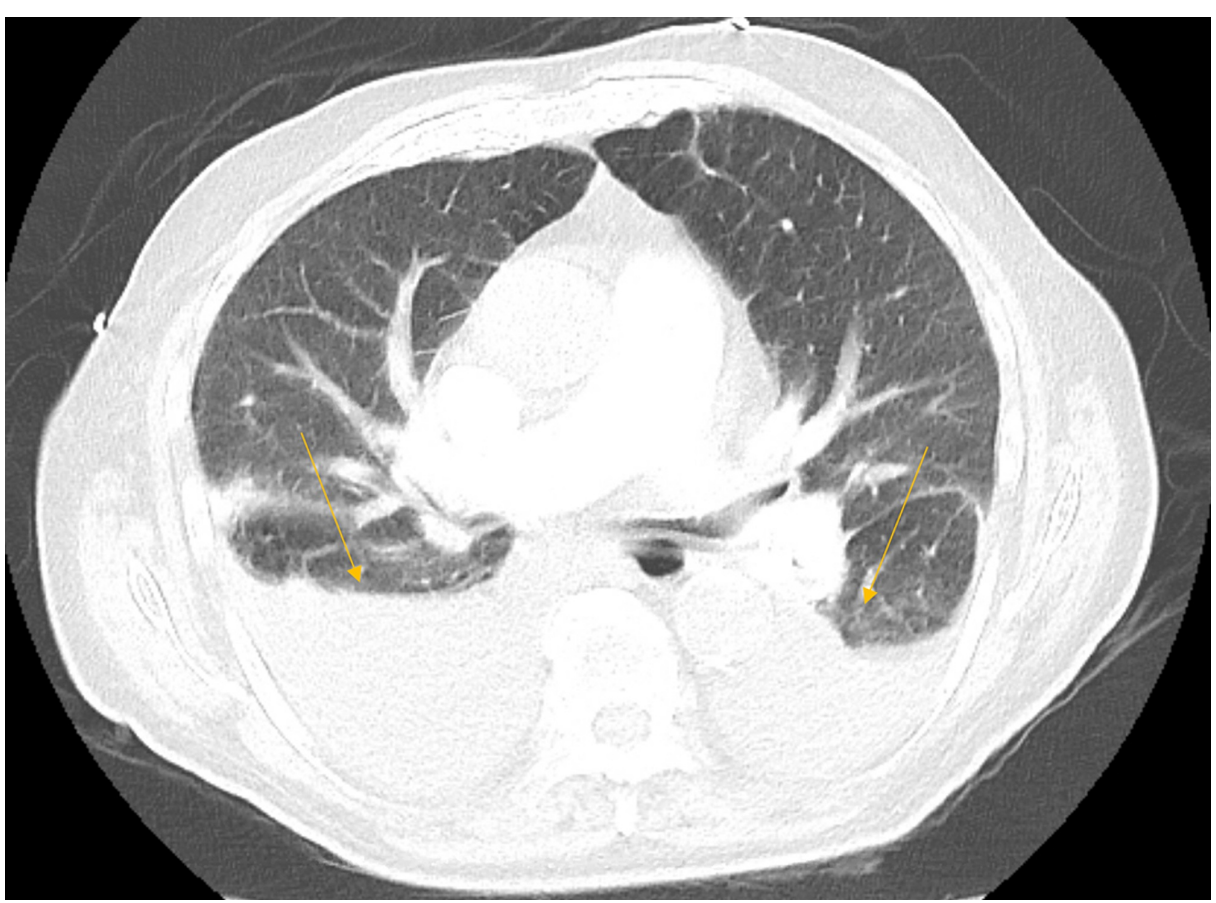


FIGURE 2: CT angiography revealed bilateral pleural effusions (orange arrows) with no signs of any inflammatory process and no pulmonary embolism.

\section{Discussion}

The etiology of ALL is currently unknown. The existing hypotheses point toward pathogenesis related to ionizing radiation and/or currently unidentified infections [4]. Familial ALL, although a rare phenomenon, has been associated with inherited mutations of TP53, PAX5, and ETV6 [5,6]. Studies have also shown an increased incidence of B-ALL in patients with Down syndrome [7]. Certain single nucleotide polymorphisms (GATA3, ARID5B, IKZF1, CEBPE, CDKN2A/B) have also been associated with ALL [8,9]. The identification of the $\mathrm{BCR} / \mathrm{ABL}$ fusion chromosome (Philadelphia chromosome) is also important in order to determine cases of BCR/ABL positive ALL [10]. The Philadelphia chromosome is detected and approximately $25 \%$ of adult ALL. It is very rare in pediatric cases, occurring in only $2-4 \%$ of cases. The incidence of the mutation increases with age, occurring in 20-30\% of ALL patients older than 60 years old [11-13]. The Philadelphia chromosome in ALL used to be an adverse prognostic indicator, however, given the advent of tyrosine kinase inhibitor therapy, its prognostic contribution is currently unclear [14].

Flow cytometry is essential for the diagnosis of ALL. The immunophenotype needs to be defined by flow cytometry and/or immunohistochemistry. The material can be circulating lymphoblasts, bone marrow biopsy, or lymph nodes. Cytogenetic analysis consisting of conventional karyotype with chromosomal banding, with or without fluorescence in situ hybridization (FISH), along with molecular analysis for determination of gene expression patterns is part of the diagnostic evaluation of ALL. It should be performed on either the circulating lymphoblasts, the bone marrow biopsy specimen, or the lymph node for classification purposes.

Dasatinib is a multitargeted tyrosine kinase inhibitor with significant activity in Philadephia positive ALL resistant to imatinib. It has also shown significant result in treatment-naïve patients $[15,16]$. It has activity against many $\mathrm{BCR} / \mathrm{ABL}$ kinase domain mutations that are resistant to imatinib and may be present at low levels at the time of Philadelphia positive ALL diagnosis [17,18]. In combination with chemotherapy, dasatinib results in complete remission rates of approximately 90 to 95 percent [15,19]. Common side effects include myelosuppression and platelet dysfunction. Pleural effusions, either unilateral or bilateral, have been reported with the use of dasatinib, with reported percentages being in the range of $15-30 \%$ of cases [20]. QT segment prolongation has been noted. Of note, contrary to imatinib, dasatinib penetrates the blood-brain barrier. In our case, the presence of bilateral pleural effusions in the absence of other apparent etiology should highlight the importance of keeping in mind dasatinib as a potential causative agent.

\section{Conclusions}

In our case, the patient presented with a clinical picture concerning acute hypoxic respiratory failure secondary to infectious etiology. Extensive infectious workup was negative. Heart failure evaluation was also unrevealing. Our heightened awareness about the adverse effect profile of dasatinib assisted with the prompt and appropriate diagnosis of the cause of the patient's symptomatology and led to the resolution of the patient's symptoms upon discontinuation of the medication.

\section{Additional Information}

\section{Disclosures}

Human subjects: Consent was obtained by all participants in this study. Conflicts of interest: In compliance with the ICMJE uniform disclosure form, all authors declare the following: Payment/services info: All authors have declared that no financial support was received from any organization for the submitted work. Financial relationships: All authors have declared that they have no financial relationships at present or within the previous three years with any organizations that might have an interest in the submitted work. Other relationships: All authors have declared that there are no other relationships or activities that could appear to have influenced the submitted work.

\section{References}

1. Dores GM, Devesa SS, Curtis RE, Linet MS, Morton LM: Acute leukemia incidence and patient survival among children and adults in the United States, 2001-2007. Blood. 2012, 119:34-43. 10.1182/blood-2011-04347872

2. Redaelli A, Laskin BL, Stephens JM, Botteman MF, Pashos CL: A systematic literature review of the clinical and epidemiological burden of acute lymphoblastic leukaemia (ALL). Eur J Cancer Care (Engl). 2005, 14:5362. 10.1111/j.1365-2354.2005.00513.x

3. Laurini JA, Perry AM, Boilesen E, et al.: Classification of non-Hodgkin lymphoma in Central and South America: a review of 1028 cases. Blood. 2012, 120:4795-4801. 10.1182/blood-2012-07-440073 
4. Inaba H, Greaves M, Mullighan CG: Acute lymphoblastic leukaemia. Lancet. 2013, 381:1943-1955. 10.1016/s0140-6736(12)62187-4

5. Shah S, Schrader KA, Waanders E, et al.: A recurrent germline PAX5 mutation confers susceptibility to pre-B cell acute lymphoblastic leukemia. Nat Genet. 2013, 45:1226-1231. 10.1038/ng.2754

6. Powell BC, Jiang L, Muzny DM, et al.: Identification of TP53 as an acute lymphocytic leukemia susceptibility gene through exome sequencing. Pediatr Blood Cancer. 2013, 60:1-3. 10.1002/pbc.24417

7. Ziino O, Rondelli R, Micalizzi C, Luciani M, Conter V, Aricò M: Acute lymphoblastic leukemia in children with associated genetic conditions other than Down's syndrome. The AIEOP experience. Haematologica. 2006, 91:139-140.

8. Papaemmanuil E, Hosking FJ, Vijayakrishnan J, et al.: Loci on 7p12.2, 10q21.2 and 14q11.2 are associated with risk of childhood acute lymphoblastic leukemia. Nat Genet. 2009, 41:1006-1010. 10.1038/ng.430

9. Perez-Andreu V, Roberts KG, Harvey RC, et al.: Inherited GATA3 variants are associated with Ph-like childhood acute lymphoblastic leukemia and risk of relapse. Nat Genet. 2013, 45:1494-1498. 10.1038/ng.2803

10. Roberts KG, Li Y, Payne-Turner D, et al.: Targetable kinase-activating lesions in Ph-like acute lymphoblastic leukemia. N Engl J Med. 2014, 371:1005-1015. 10.1056/NEJMoa1403088

11. Ribeiro RC, Abromowitch M, Raimondi SC, Murphy SB, Behm F, Williams DL: Clinical and biologic hallmarks of the Philadelphia chromosome in childhood acute lymphoblastic leukemia. Blood. 1987, 70:948-953.

12. Moorman AV, Chilton L, Wilkinson J, Ensor HM, Bown N, Proctor SJ: A population-based cytogenetic study of adults with acute lymphoblastic leukemia. Blood. 2010, 115:206-214. 10.1182/blood-2009-07-232124

13. Pui CH, Robison LL, Look AT: Acute lymphoblastic leukaemia. Lancet. 2008, 371:1030-1043. 10.1016/s01406736(08)60457-2

14. Schultz KR, Carroll A, Heerema NA, et al.: Long-term follow-up of imatinib in pediatric Philadelphia chromosome-positive acute lymphoblastic leukemia: Children's Oncology Group study AALL0031. Leukemia. 2014, 28:1467-1471. 10.1038/leu.2014.30

15. Ravandi F, O'Brien S, Thomas D, et al.: First report of phase 2 study of dasatinib with hyper-CVAD for the frontline treatment of patients with Philadelphia chromosome-positive $(\mathrm{Ph}+)$ acute lymphoblastic leukemia. Blood. 2010, 116:2070-2077. 10.1182/blood-2009-12-261586

16. Ottmann O, Dombret H, Martinelli G, et al.: Dasatinib induces rapid hematologic and cytogenetic responses in adult patients with Philadelphia chromosome positive acute lymphoblastic leukemia with resistance or intolerance to imatinib: interim results of a phase 2 study. Blood. 2007, 110:2309-2315. 10.1182/blood2007-02-073528

17. Soverini S, Vitale A, Poerio A, et al.: Philadelphia-positive acute lymphoblastic leukemia patients already harbor BCR-ABL kinase domain mutations at low levels at the time of diagnosis. Haematologica. 2011, 96:552-557. 10.3324/haematol.2010.034173

18. Pfeifer H, Wassmann B, Pavlova A, et al.: Kinase domain mutations of BCR-ABL frequently precede imatinib-based therapy and give rise to relapse in patients with de novo Philadelphia-positive acute lymphoblastic leukemia (Ph+ ALL). Blood. 2007, 110:727-734. 10.1182/blood-2006-11-052373

19. Ravandi F, O'Brien SM, Cortes JE, et al.: Long-term follow-up of a phase 2 study of chemotherapy plus dasatinib for the initial treatment of patients with Philadelphia chromosome-positive acute lymphoblastic leukemia. Cancer. 2015, 121:4158-4164. 10.1002/cncr.29646

20. Latagliata R, Breccia M, Fava C, et al.: Incidence, risk factors and management of pleural effusions during dasatinib treatment in unselected elderly patients with chronic myelogenous leukaemia. Hematol Oncol. 2013, 31:103-109. 